\title{
Strict global minimizers and higher-order generalized strong invexity in multiobjective optimization
}

\author{
Guneet Bhatia ${ }^{1 *}$ and Rishi Rajan Sahay ${ }^{2}$
}

\section{"Correspondence:}

guneet172@yahoo.co.in

1 Department of Mathematics,

University of Delhi, Delhi, 110007,

India

Full list of author information is

available at the end of the article

\begin{abstract}
Higher-order strict minimizers with respect to a nonlinear function for a multiobjective optimization problem are introduced and are characterized via sufficient optimality conditions and higher-order mixed saddle points of a vector-valued partial Lagrangian. To this aim, we present certain generalizations of higher-order strong invexity. A mixed dual is proposed and corresponding duality results are obtained. An equivalent optimization problem for the given multiobjective optimization problem is introduced. It is shown that the problem of finding higher-order strict minimizers with respect to a nonlinear function for the given problem reduces to that of finding strict minimizers in the ordinary sense for an equivalent problem.

MSC: $26 \mathrm{~A} 51 ; 90 \mathrm{C} 29 ; 90 \mathrm{C} 46$
\end{abstract}

Keywords: higher-order strong invexity; strict minimizer of order $m$; partial Lagrangian; mixed saddle point

\section{Introduction}

Multiobjective optimization problems occupy an important place in the theory of optimization. Several solution concepts for multiobjective optimization problem have appeared in the literature viz. efficiency, weak efficiency and proper efficiency $[1,2]$. The concept of higher-order local minimizer plays an important role in the convergence analysis of iterative numerical methods [3] and in stability results [4]. For a scalar optimization problem, Auslender [5] derived necessary and sufficient optimality conditions for isolated local minima of order 1 and 2, and Ward [6] presented the notion of strict local minimum of order $m$. Jimenez [7] extended the idea of Ward [6] to define the notion of a strict local efficient solution of order $m$ for a vector minimization problem. Bhatia [8] extended the notion of Ward to define the higher-order global strict minimizer for a multiobjective optimization problem. Sahay and Bhatia [9] introduced the notion of a strict minimizer of order $m$ with respect to a nonlinear function for a scalar optimization problem.

In this paper, we move a step ahead in this direction and introduce the concept of a higher-order strict minimizer with respect to a nonlinear function for a multiobjective optimization problem. For the purpose of studying this new solution concept, we present certain generalizations of higher-order strong invexity [9]. Sufficient optimality conditions characterizing this solution concept are obtained. A mixed dual is proposed and wellknown duality results are established. A partial vector-valued Lagrangian for the multi-

\section{焦 Springer}

(c) 2013 Bhatia and Sahay; licensee Springer. This is an Open Access article distributed under the terms of the Creative Commons Attribution License (http://creativecommons.org/licenses/by/2.0), which permits unrestricted use, distribution, and reproduction in any medium, provided the original work is properly cited. 
objective optimization problem is introduced. Higher-order mixed saddle points for the partial Lagrangian with respect to a nonlinear function are shown to be equivalent to the higher-order strict minimizers with respect to the same function. Further, an equivalent optimization problem that enables one to find the higher-order strict minimizers for a given multiobjective optimization problem in a simpler manner is presented.

\section{Higher-order global strict minimizers}

In this paper, we study the following multiobjective optimization problem:

$$
\begin{array}{ll}
(\mathrm{MOP}) & \operatorname{minimize} f(x)=\left(f_{1}(x), \ldots, f_{p}(x)\right) \\
& \text { subject to } g_{j}(x) \leq 0, \quad j=1,2, \ldots, q,
\end{array}
$$

where $f_{i}, g_{j}: X \rightarrow R, i=1,2, \ldots, p, j=1,2, \ldots, q$ are real-valued differentiable functions and $X$ is a non-empty open subset of $R^{n}$ endowed with the Euclidean norm $\|\cdot\|$.

We denote by $S=\left\{x \in X: g_{j}(x) \leq 0, j=1,2, \ldots, q\right\}$ the set of all feasible solutions for (MOP) and let $I(x)=\left\{j: g_{j}(x)=0\right\}$ be the set of indices corresponding to active constraints. Let $B\left(x^{0}, \varepsilon\right)=\left\{x \in R^{n}:\left\|x-x^{0}\right\|<\varepsilon\right\}$ denote an open ball with centre $x^{0}$ and radius $\varepsilon$.

Definition 2.1 ([7]) A point $x^{0} \in S$ is a strict local minimizer for (MOP) if there exists an $\varepsilon>0$ such that

$$
f(x) \nless f\left(x^{0}\right) \text { for all } x \in B\left(x^{0}, \varepsilon\right) \cap S \text {, }
$$

that is, there exists no $x \in B\left(x^{0}, \varepsilon\right) \cap S$ such that

$$
f(x)<f\left(x^{0}\right) .
$$

Definition 2.2 ([8]) Let $m \geq 1$ be an integer. A point $x^{0} \in S$ is a local strict minimizer of order $m$ for (MOP) if there exists an $\varepsilon>0$ and a constant $c \in \operatorname{int} R_{+}^{p}$ such that

$$
f(x) \nless f\left(x^{0}\right)+c\left\|x-x^{0}\right\|^{m} \quad \text { for all } x \in B\left(x^{0}, \varepsilon\right) \cap S .
$$

The notion of a local strict minimizer reduces to the global sense if the ball $B\left(x^{0}, \varepsilon\right)$ is replaced by the whole space $R^{n}$.

The following example illustrates that in some cases $x^{0}$ may fail to be a strict minimizer in the sense of the above definition.

Example 2.1 Let $S=[0,1]$ and $f(x)=\left(x^{3}, \sin ^{3} x\right)$, then $x^{0}=0$ is not a strict minimizer of order 1 in the sense of Definition 2.2, since for any $c=\left(c_{1}, c_{2}\right) \in \operatorname{int} R_{+}^{2}$, there exists an $x$ satisfying $0<x<c_{1}^{1 / 2}, 0<\frac{\sin ^{3} x}{x}<c_{2}$ such that $f(x)<f\left(x^{0}\right)+c\left\|x-x^{0}\right\|$.

The above example motivates us to introduce a new notion of a strict minimizer of order $m$ with respect to a nonlinear function for the multiobjective optimization problem (MOP). 
Definition 2.3 Let $m \geq 1$ be an integer. A point $x^{0} \in S$ is a local strict minimizer of order $m$ for (MOP) with respect to a nonlinear function $\psi: S \times S \rightarrow R^{n}$, if there exists an $\varepsilon>0$ and a constant $c \in \operatorname{int} R_{+}^{p}$ such that

$$
f(x) \nless f\left(x^{0}\right)+c\left\|\psi\left(x, x^{0}\right)\right\|^{m} \quad \text { for all } x \in B\left(x^{0}, \varepsilon\right) \cap S .
$$

Definition 2.4 Let $m \geq 1$ be an integer. A point $x^{0} \in S$ is a strict minimizer of order $m$ for (MOP) with respect to a nonlinear function $\psi: S \times S \rightarrow R^{n}$ if there exists a constant $c \in \operatorname{int} R_{+}^{p}$ such that

$$
f(x) \nless f\left(x^{0}\right)+c\left\|\psi\left(x, x^{0}\right)\right\|^{m} \quad \text { for all } x \in S \text {. }
$$

Remark 2.1 The function $\psi$ plays an important role in the notion of a strict minimizer defined above. For the problem considered in Example 2.1, $x^{0}=0$ failed to be a strict minimizer of order 1 in the usual sense; however, it is important to observe here that $x^{0}=0$ is a strict minimizer of order 1 with respect to $\psi\left(x, x^{0}\right)=\sin ^{3} x-\sin ^{3} x^{0}$ for $c=(1,1)$.

Remark 2.2 The study of higher-order minimizers is pertinent as these minimizers play an important role in the convergence analysis of iterative numerical methods and in stability results. These minimizers are often exactly those satisfying an $m$ th derivative test $[6,7]$. It is clear that any strict minimizer of order $m$ is also a strict minimizer for (MOP). Converse of this statement may not be true. If $x^{0}$ is a strict minimizer of order $m$ with respect to a nonlinear function $\psi$, then it is also a strict minimizer of order $j$ with respect to the same $\psi$ for all $j>m$.

We recall that [1] a set $S \subseteq R^{n}$ is invex with respect to $\eta$ if there exists $\eta: S \times S \rightarrow R^{n}$ such that for all $x, y \in S$ and all $\lambda \in[0,1], y+\lambda \eta(x, y) \in S$. Throughout this paper, we assume $S \subseteq X$ to be an invex set.

Definition 2.5 ([9]) A differentiable function $f: X \rightarrow R$ is said to be strongly invex of order $m \geq 1$ with respect to $\eta, \psi$ on $S$ if there exists a constant $c>0$ such that for all $x, y \in S$,

$$
f(x)-f(y) \geq \nabla f(y)^{t} \eta(x, y)+c\|\psi(x, y)\|^{m} .
$$

If $\psi(x, y)=0$, then the above definition reduces to the notion of invexity. If $\psi(x, y)=x-y$, $\eta(x, y)=x-y$, the definition reduces to the definition of strong convexity of order $m$ [10].

Remark 2.3 It is important to observe that there exist functions which are strongly invex of order $m$ but are not strongly convex of any order. For example, let $X=R^{2}, S=\left\{\left(x_{1}, x_{2}\right) \in\right.$ $\left.R^{2}: 0 \leq x_{1}, x_{2} \leq 1\right\}, f(x)=x_{1}+x_{2}^{2}, \eta(x, y)=\left(-y_{1},-y_{2}\right)$ and $\psi(x, y)=\left(x_{1} / \sqrt{1+y_{2}}, 0\right)$, where $x=\left(x_{1}, x_{2}\right)^{t}$ and $y=\left(y_{1}, y_{2}\right)^{t}$. Then, for all $x, y \in S$ and $\lambda \in[0,1]$, we have $y+\lambda \eta(x, y)=\left(y_{1}(1-\right.$ $\left.\lambda), y_{2}(1-\lambda)\right) \in S$, thus $S$ is an invex set with respect to $\eta$. Clearly, $f$ is strongly invex of order $m \geq 1$ with respect to $\eta$ and $\psi$ as defined above for $0<c \leq 1$. However, on choosing $x=(1,1 / 2)$ and $y=(0,1 / 2)$, it is evident that $f$ is not strongly convex of any order for any $c>0$. 
Remark 2.4 Every strongly invex function of order $m$ with respect to $\eta$ and $\psi$ is invex. However, converse of this statement may not be true [9].

We now present the following generalizations of higher-order strong invexity.

Definition 2.6 A differentiable function $f: X \rightarrow R$ is said to be strongly pseudoinvex type I of order $m$ with respect to $\eta, \psi$ on $S$ if there exists a constant $c>0$ such that for all $x, y \in S$,

$$
\nabla f(y)^{t} \eta(x, y) \geq 0 \quad \text { implies } \quad f(x) \geq f(y)+c\|\psi(x, y)\|^{m},
$$

or equivalently, $f(x)<f(y)+c\|\psi(x, y)\|^{m}$ implies $\nabla f(y)^{t} \eta(x, y)<0$.

Remark 2.5 Strong invexity of order $m$ with respect to $\eta$ and $\psi$ implies strong pseudoinvexity type I of order $m$ with respect to the same $\eta$ and $\psi$. However, converse is not true in general. For example, let $X=R, S=(0, \pi / 2], f(x)=1+\cos x, \eta(x, y)=\frac{(\cos y-\cos x)}{\sin y}$ and $\psi(x, y)=\cos x-\cos y$, then for $c=1 / 2^{m-1}, f$ is strongly pseudoinvex type I of order $m \geq 1$ with respect to $\eta$ and $\psi$ on $S$ but is not strongly invex of any order $m$ with respect to these $\eta$ and $\psi$.

Definition 2.7 A differentiable function $f: X \rightarrow R$ is said to be strongly pseudoinvex type II of order $m$ with respect to $\eta, \psi$ on $S$ if there exists a constant $c>0$ such that for all $x, y \in S$,

$$
\nabla f(y)^{t} \eta(x, y)+c\|\psi(x, y)\|^{m} \geq 0 \text { implies } f(x) \geq f(y) .
$$

Definition 2.8 A differentiable function $f: X \rightarrow R$ is said to be strongly quasiinvex type I of order $m$ with respect to $\eta, \psi$ on $S$ if there exists a constant $c>0$ such that for all $x, y \in S$,

$$
f(x) \leq f(y) \quad \text { implies } \quad \nabla f(y)^{t} \eta(x, y)+c\|\psi(x, y)\|^{m} \leq 0 .
$$

Definition 2.9 A differentiable function $f: X \rightarrow R$ is said to be strongly quasiinvex type II of order $m$ with respect to $\eta, \psi$ on $S$ if there exists a constant $c>0$ such that for all $x, y \in S$,

$$
f(x) \leq f(y)+c\|\psi(x, y)\|^{m} \quad \text { implies } \quad \nabla f(y)^{t} \eta(x, y) \leq 0 .
$$

The relations between these classes of functions and some related classes are summarized in Figure 1 (note: it is important to observe that there is no relation between type II functions and corresponding notions of type I functions presented in Figure 1).

\section{Local-global property and optimality conditions}

Theorem 3.1 Suppose $x^{0} \in S$ is a strict local minimizer of order $m$ with respect to $\psi$ for (MOP) and the functions $f_{i}: X \rightarrow R, i=1,2, \ldots, p$ are strongly pseudoinvex type I of order $m$ with respect to the same $\eta$ and $\psi$ on $S$. Then $x^{0}$ is a strict minimizer of order $m$ with respect to the same $\psi$ for (MOP).

Proof Since $x^{0} \in S$ is a local strict minimizer of order $m$ with respect to $\psi$ for (MOP), therefore there exists an $\varepsilon>0$ and a constant $c=\left(c_{1}, \ldots, c_{p}\right) \in \operatorname{int} R_{+}^{p}$ such that

$$
f_{i}(x) \nless f_{i}\left(x^{0}\right)+c_{i}\left\|\psi\left(x, x^{0}\right)\right\|^{m}, \quad i=1,2, \ldots, p \text {, for all } x \in B\left(x^{0}, \varepsilon\right) \cap S .
$$




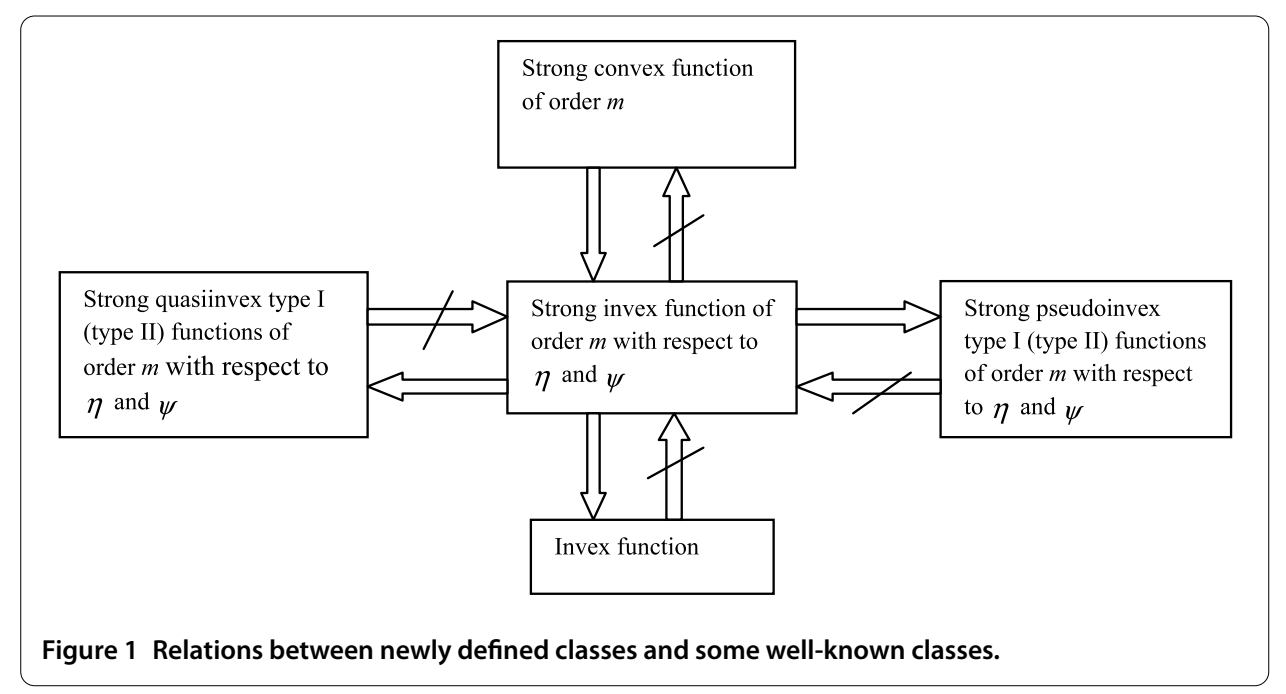

Let us suppose that $x^{0}$ is not a strict minimizer of order $m$ with respect to $\psi$ for (MOP), then for all $c_{i}>0, i=1,2, \ldots, p$, there exists some $z \in S$ such that

$$
f_{i}(z)<f_{i}\left(x^{0}\right)+c_{i}\left\|\psi\left(z, x^{0}\right)\right\|, \quad i=1,2, \ldots, p
$$

For $x^{0} \in S$ and sufficiently small $\lambda \in(0,1)$ and $\eta: S \times S \rightarrow R^{n}$, we have $x^{0}+\lambda \eta\left(z, x^{0}\right) \in$ $B\left(x^{0}, \varepsilon\right) \cap S$.

As $f_{i}, i=1,2, \ldots, p$ are strongly pseudoinvex type I of order $m$ on $S$ with respect to $\eta$ and $\psi$ for $z, x^{0} \in S$, it follows from the set of above inequalities that

$$
\nabla f_{i}\left(x^{0}\right)^{t} \eta\left(z, x^{0}\right)<0, \quad i=1,2, \ldots, p
$$

Thus, there exists $\sigma>0$ such that for $0<\lambda \leq \sigma, f_{i}\left(x^{0}+\lambda \eta\left(z, x^{0}\right)\right)<f_{i}\left(x^{0}\right), i=1,2, \ldots, p$, which implies that for all $c_{i}>0, i=1,2, \ldots, p$ and for every nonlinear function $\psi$, we have

$$
f_{i}\left(x^{0}+\lambda \eta\left(z, x^{0}\right)\right)<f_{i}\left(x^{0}\right)+c_{i}\left\|\psi\left(x^{0}+\lambda \eta\left(z, x^{0}\right), x^{0}\right)\right\|^{m}, \quad i=1,2, \ldots, p ; 0<\lambda \leq \sigma .
$$

This contradicts (3.1).

Theorem 3.2 (Fritz John type necessary optimality conditions) Suppose $x^{0}$ is a strict minimizer of order $m$ with respect to a nonlinear function $\psi: S \times S \rightarrow R^{n}$ for (MOP) and the functions $f_{i}, i=1,2, \ldots, p, g_{j}, j=1,2, \ldots, q$ are differentiable at $x^{0}$. Then there exists $\lambda_{i}^{0} \geq 0$, $i=1,2, \ldots, p, \mu_{j}^{0} \geq 0, j=1,2, \ldots, q$ such that

$$
\begin{aligned}
& \sum_{i=1}^{p} \lambda_{i}^{0} \nabla f_{i}\left(x^{0}\right)+\sum_{j=1}^{q} \mu_{j}^{0} \nabla g_{j}\left(x^{0}\right)=0, \\
& \mu_{j}^{0} g_{j}\left(x^{0}\right)=0, \quad j=1,2, \ldots, q, \\
& \left(\lambda^{0}, \mu^{0}\right) \neq 0 .
\end{aligned}
$$

Definition 3.1 (MOP) is said to satisfy Slater's constraint qualification (SCQ) at $x^{0}$ if there exists $\bar{x} \in X$ such that $g_{j}(\bar{x})<0, j \in I\left(x^{0}\right)$. 
Theorem 3.3 (Karush-Kuhn-Tucker type necessary optimality conditions) Suppose $x^{0}$ is a strict minimizer of order $m$ with respect to a nonlinear function $\psi: S \times S \rightarrow R^{n}$ for (MOP) and the functions $f_{i}, i=1,2, \ldots, p, g_{j}, j=1,2, \ldots, q$ are differentiable at $x^{0}$. Assume that (SCQ) holds at $x^{0}$, then there exist $\lambda_{i}^{0} \geq 0, i=1,2, \ldots, p, \mu_{j}^{0} \geq 0, j=1,2, \ldots, q$ such that

$$
\begin{aligned}
& \sum_{i=1}^{p} \lambda_{i}^{0} \nabla f_{i}\left(x^{0}\right)+\sum_{j=1}^{q} \mu_{j}^{0} \nabla g_{j}\left(x^{0}\right)=0, \\
& \mu_{j}^{0} g_{j}\left(x^{0}\right)=0, \quad j=1,2, \ldots, q, \\
& \lambda^{0 t} \cdot e=1, \quad \text { where } e=(1, \ldots, 1) \in R^{p} .
\end{aligned}
$$

Theorem 3.4 (Sufficient optimality conditions) Let the conditions (3.2)-(3.4) be satisfied at $x^{0} \in S$. Suppose $f_{i}, i=1,2, \ldots$, p are strongly pseudoinvex type I of order $m$ and $g_{j}, j \in I\left(x^{0}\right)$ are strongly quasiinvex type I of order $m$ with respect to the same $\eta$ and $\psi$ on $S$. Then $x^{0}$ is a strict minimizer of order $m$ with respect to $\psi$ for (MOP).

Proof On the contrary, suppose that $x^{0} \in S$ is not a strict minimizer of order $m$ with respect to $\psi$ for (MOP). Then, for $\bar{c}_{i}>0, i=1,2, \ldots, p$, there exists some $\bar{x} \in S$ such that

$$
f_{i}(\bar{x})<f_{i}\left(x^{0}\right)+\bar{c}_{i}\left\|\psi\left(\bar{x}, x^{0}\right)\right\|^{m}, \quad i=1,2, \ldots, p .
$$

As $f_{i}, i=1,2, \ldots, p$ are strongly pseudoinvex type I of order $m$ with respect to $\eta$ and $\psi$ on $S$, therefore, from (3.5), we have

$$
\nabla f_{i}\left(x^{0}\right)^{t} \eta\left(\bar{x}, x^{0}\right)<0, \quad i=1,2, \ldots, p .
$$

As $\lambda_{i}^{0} \geq 0, i=1,2, \ldots, p$, and $\lambda^{0 t} \cdot e=1$, the above system of inequalities reduces to

$$
\sum_{i=1}^{p} \lambda_{i}^{0} \nabla f_{i}\left(x^{0}\right)^{t} \eta\left(\bar{x}, x^{0}\right)<0
$$

Now, for $\bar{x} \in S, g_{j}(\bar{x}) \leq g_{j}\left(x^{0}\right), j \in I\left(x^{0}\right)$. As $g_{j}, j \in I\left(x^{0}\right)$, is strongly quasiinvex type I of order $m$ with respect to the same $\eta$ and $\psi$ on $S$, it follows that there exist constants $\beta_{j}>0, j \in I\left(x^{0}\right)$ such that

$$
\nabla g_{j}\left(x^{0}\right)^{t} \eta\left(\bar{x}, x^{0}\right)+\beta_{j}\left\|\psi\left(\bar{x}, x^{0}\right)\right\|^{m} \leq 0 .
$$

Further, since $\mu_{j}^{0} \geq 0, j \in I\left(x^{0}\right)$, it follows from the above relation that

$$
\sum_{j \in I\left(x^{0}\right)} \mu_{j}^{0} \nabla g_{j}\left(x^{0}\right)^{t} \eta\left(\bar{x}, x^{0}\right)+\sum_{j \in I\left(x^{0}\right)} \mu_{j}^{0} \beta_{j}\left\|\psi\left(\bar{x}, x^{0}\right)\right\|^{m} \leq 0
$$

As $\mu_{j}^{0}=0$, for $j \notin I\left(x^{0}\right)$, we have

$$
\sum_{j=1}^{q} \mu_{j}^{0} \nabla g_{j}\left(x^{0}\right)^{t} \eta\left(\bar{x}, x^{0}\right)+\sum_{j=1}^{q} \mu_{j}^{0} \beta_{j}\left\|\psi\left(\bar{x}, x^{0}\right)\right\|^{m} \leq 0 .
$$


Adding (3.6) and (3.7), we obtain

$$
\eta^{t}\left(\bar{x}, x^{0}\right)\left[\sum_{i=1}^{p} \lambda_{i}^{0} \nabla f_{i}\left(x^{0}\right)+\sum_{j=1}^{q} \mu_{j}^{0} \nabla g_{j}\left(x^{0}\right)\right]+\sum_{j=1}^{q} \mu_{j}^{0} \beta_{j}\left\|\psi\left(\bar{x}, x^{0}\right)\right\|^{m}<0 .
$$

On using (3.2), we have $\sum_{j=1}^{q} \mu_{j}^{0} \beta_{j}\left\|\psi\left(\bar{x}, x^{0}\right)\right\|^{m}<0$, which is not possible.

Remark 3.1 The result of the above theorem also holds under the conditions that $f_{i}, i=$ $1,2, \ldots, p$ are strongly invex of order $m$ with respect to the same $\eta$ and $\psi$ and $g_{j}, j \in I\left(x^{0}\right)$ are strongly quasiinvex type II of order $m$ with respect to the same $\eta$ and $\psi$ on $S$.

\section{Duality}

In this section, we develop duality relationship between (MOP) and its mixed dual (MD) under the assumption of generalized strong invexity of order $m$ with respect to a nonlinear function.

Let the index set $Q=\{1,2, \ldots, q\}$ be partitioned into two disjoint subsets $J$ and $K$ such that $Q=J \cup K$. The mixed dual for (MOP) is given by

$$
\begin{aligned}
& \text { (MD) maximize } f(u)+\mu_{J} g_{J}(u) e \\
& \text { subject to } \sum_{i=1}^{p} \lambda_{i} \nabla f_{i}(u)+\sum_{j=1}^{q} \mu_{j} \nabla g_{j}(u)=0, \\
& \mu_{j} g_{j}(u) \geq 0, \quad j \in K, \\
& \lambda_{i} \geq 0, \quad i=1,2, \ldots, p, \quad \lambda^{t} \cdot e=1, \quad e=(1, \ldots, 1) \in R^{p}, \\
& \mu_{j} \geq 0, \quad j=1,2, \ldots, q .
\end{aligned}
$$

Theorem 4.1 (Weak duality) Let $x$ and $(u, \lambda, \mu)$ be feasible for (MOP) and (MD) respectively. Suppose $f_{i}+\mu_{J} g_{J}, i=1,2, \ldots, p$ are strongly pseudoinvex type I of order $m$ and $\mu_{j} g_{j}$, $j \in K$ is strongly quasiinvex type II of order $m$ with respect to the same $\eta$ and $\psi$, then there exists $c \in \operatorname{int} R_{+}^{p}$ such that

$$
f(x) \nless f(u)+\mu_{J} g_{J}(u) e+c\|\psi(x, u)\|^{m} .
$$

Proof Suppose on the contrary, for every $c \in \operatorname{int} R_{+}^{p}$, we have

$$
f(x)<f(u)+\mu_{J} g_{J}(u) e+c\|\psi(x, u)\|^{m}
$$

or

$$
f_{i}(x)<f_{i}(u)+\mu_{J} g_{J}(u)+c_{i}\|\psi(x, u)\|^{m}, \quad i=1,2, \ldots, p .
$$

Since $x$ is feasible for (MOP) and $\mu_{j} \geq 0$, therefore for $i=1,2, \ldots, p$, we have

$$
f_{i}(x)+\mu_{J} g_{J}(x)<f_{i}(u)+\mu_{J} g_{J}(u)+c_{i}\|\psi(x, u)\|^{m}
$$


Using strong pseudoinvexity type I of order $m$ for $f_{i}+\mu_{J} g_{J}, i=1,2, \ldots, p$, with respect to $\eta$ and $\psi$, we have

$$
\eta^{t}(x, u)\left[\nabla f_{i}(u)+\sum_{j \in J} \mu_{j} \nabla g_{j}(u)\right]<0, \quad i=1,2, \ldots, p .
$$

The above set of inequalities along with $\lambda_{i} \geq 0, i=1,2, \ldots, p$ and (4.3) yields

$$
\eta^{t}(x, u)\left[\sum_{i=1}^{p} \lambda_{i} \nabla f_{i}(u)+\sum_{j \in J} \mu_{j} \nabla g_{j}(u)\right]<0
$$

Now, for $x \in S, g_{j}(x) \leq 0$, and since $\mu_{j} \geq 0$, we have $\mu_{j} g_{j}(x) \leq 0, j \in K$. Moreover, $(u, \lambda, \mu)$ is feasible for (MD), therefore

$$
\mu_{j} g_{j}(x) \leq \mu_{j} g_{j}(u), \quad j \in K
$$

or

$$
\mu_{j} g_{j}(x) \leq \mu_{j} g_{j}(u)+\beta_{j}\|\psi(x, u)\|^{m} \quad \text { for } \beta_{j}>0, j \in K \text {. }
$$

Since $\mu_{j} g_{j}, j \in K$ is strongly quasiinvex type II of order $m$ with respect to $\eta$ and $\psi$, therefore

$$
\eta^{t}(x, u) \mu_{j} \nabla g_{j}(u) \leq 0, \quad j \in K,
$$

which further implies that

$$
\eta^{t}(x, u) \sum_{j \in K} \mu_{j} \nabla g_{j}(u) \leq 0
$$

Adding (4.4) and (4.5), we have

$$
\eta^{t}(x, u)\left[\sum_{i=1}^{p} \lambda_{i} \nabla f_{i}(u)+\sum_{j=1}^{q} \mu_{j} \nabla g_{j}(u)\right]<0 .
$$

This contradicts (4.1).

Theorem 4.2 (Strong duality) Suppose $x^{0}$ is a strict minimizer of order $m$ with respect to a nonlinear function $\psi: S \times S \rightarrow R^{n}$ for (MOP). Assume that (SCQ) holds at $x^{0}$, then there exist $\lambda_{i}^{0} \geq 0, i=1,2, \ldots, p$ and $\mu_{j}^{0} \geq 0, j=1,2, \ldots, q$ such that $\left(x^{0}, \lambda^{0}, \mu^{0}\right)$ is feasible for (MD). Further, if the conditions of Theorem 4.1 hold, then $\left(x^{0}, \lambda^{0}, \mu^{0}\right)$ is a strict maximizer of order $m$ for (MD).

Proof The proof follows from Theorem 3.3 and Theorem 4.1.

\section{Partial vector Lagrangian and mixed saddle point}

The saddle point of the Lagrangian is always a global minimizer for the inequality constrained minimization problem. Due to the significance of this result in economics and 
optimization theory, several researchers $[1,2,11]$ have obtained the equivalence between the saddle point and optimal solutions of an optimization problem under various conditions on the functions involved. In this section, we define higher-order mixed saddle points with respect to a nonlinear function $\psi: S \times S \rightarrow R^{n}$ for a partial vector-valued Lagrangian of a multiobjective optimization problem. The equivalence of these saddle points and the higher-order strict minimizers with respect to the same function $\psi$ for (MOP) is established under generalized higher-order strong invexity conditions on the functions involved.

Let $Q=\{1,2, \ldots, q\}, J_{1} \subseteq Q$ and $J_{2}=Q \bigvee_{1},\left|J_{1}\right|$ denote the cardinality of index set $J_{1}$.

Definition 5.1 Vector-valued partial Lagrangian function $L: S \times R_{+}^{\left|J_{1}\right|} \rightarrow R^{p}$ for (MOP) is defined as

$$
L\left(x, \mu_{J_{1}}\right)=\left\{L_{1}\left(x, \mu_{J_{1}}\right), \ldots, L_{p}\left(x, \mu_{J_{1}}\right)\right\}
$$

where $L_{i}\left(x, \mu_{J_{1}}\right)=f_{i}(x)+\sum_{j \in J_{1}} \mu_{j} g_{j}(x), i=1,2, \ldots, p, x \in S, \mu_{J_{1}} \in R_{+}^{\left|J_{1}\right|}$.

We now introduce the notion of mixed saddle points of order $m$ with respect to a nonlinear function for (MOP) as follows.

Definition 5.2 A vector $\left(x^{0}, \mu_{J_{1}}^{0}\right) \in S \times R_{+}^{\left|J_{1}\right|}$ is said to be a mixed saddle point of order $m$ with respect to a nonlinear function $\psi$ for the partial vector-valued Lagrangian $L$ for (MOP) if there exists $c \in$ int $R_{+}^{p}$ such that

$$
\begin{aligned}
& L\left(x^{0}, \mu_{J_{1}}^{0}\right) \nless L\left(x^{0}, \mu_{J_{1}}\right) \quad \text { for all } \mu_{J_{1}} \in R_{+}^{\left|J_{1}\right|}, \\
& L\left(x, \mu_{J_{1}}^{0}\right) \nless L\left(x^{0}, \mu_{J_{1}}^{0}\right)+c\left\|\psi\left(x, x^{0}\right)\right\|^{m} \text { for all } x \in S .
\end{aligned}
$$

Theorem 5.1 Suppose that $x^{0}$ is a strict minimizer of order $m$ with respect to a nonlinear function $\psi$ for (MOP) and (SCQ) holds at $x^{0}$. Further, if $f_{i}+\sum_{j \in J_{1}} \mu_{j}^{0} g_{j}, i=1,2, \ldots, p$ are strongly pseudoinvex type I of order $m$ and $\mu_{j}^{0} g_{j}, j \in J_{2}$ is strongly quasiinvex type I of order $m$ with respect to $\eta$ and $\psi$ on $S$, then $\left(x^{0}, \mu_{J_{1}}^{0}\right)$ is a mixed saddle point of order $m$ with respect to $\psi$ for the partial Lagrangian.

Proof Suppose $x^{0}$ is a strict minimizer of order $m$ with respect to $\psi$ for (MOP) and the constraint qualification holds at $x^{0}$. Therefore, by Theorem 3.3, there exist $\lambda_{i}^{0} \geq 0, i=$ $1,2, \ldots, p$ and $\mu_{j}^{0} \geq 0, j=1,2, \ldots, q$ such that conditions (3.2)-(3.4) hold at $x^{0}$.

On the contrary, suppose that $\left(x^{0}, \mu_{J_{1}}^{0}\right)$ is not a mixed saddle point of any order for the partial vector Lagrangian function $L$ for (MOP). Then, for all $c \in \operatorname{int} R_{+}^{p}$, there exists some $\bar{x} \in S$ such that

$$
L\left(\bar{x}, \mu_{J_{1}}^{0}\right)<L\left(x^{0}, \mu_{J_{1}}^{0}\right)+c\left\|\psi\left(\bar{x}, x^{0}\right)\right\|^{m}
$$

that is,

$$
L_{i}\left(\bar{x}, \mu_{J_{1}}^{0}\right)<L_{i}\left(x^{0}, \mu_{J_{1}}^{0}\right)+c_{i}\left\|\psi\left(\bar{x}, x^{0}\right)\right\|^{m}, \quad i=1,2, \ldots, p
$$


or

$$
f_{i}(\bar{x})+\sum_{j \in J_{1}} \mu_{j}^{0} g_{j}(\bar{x})<f_{i}\left(x^{0}\right)+\sum_{j \in J_{1}} \mu_{j}^{0} g_{j}\left(x^{0}\right)+c_{i}\left\|\psi\left(\bar{x}, x^{0}\right)\right\|^{m}, \quad i=1,2, \ldots, p .
$$

Since $f_{i}+\sum_{j \in J_{1}} \mu_{j}^{0} g_{j}, i=1,2, \ldots, p$ are strongly pseudoinvex type I of order $m$ with respect to $\eta$ and $\psi$, it follows from the above inequalities that

$$
\eta^{t}\left(\bar{x}, x^{0}\right)\left[\nabla f_{i}\left(x^{0}\right)+\sum_{j \in J_{1}} \mu_{j}^{0} \nabla g_{j}\left(x^{0}\right)\right]<0, \quad i=1,2, \ldots, p
$$

Now, for $\bar{x} \in S$ and $\mu_{j}^{0} \geq 0, j \in J_{2}$, it follows that $\mu_{j}^{0} g_{j}(\bar{x}) \leq 0$. On using (3.3), we have

$$
\mu_{j}^{0} g_{j}(\bar{x}) \leq \mu_{j}^{0} g_{j}\left(x^{0}\right), \quad j \in J_{2} .
$$

Since $\mu_{j}^{0} g_{j}, j \in J_{2}$ is strongly quasiinvex type I of order $m$ on $S$ with respect to $\eta$ and $\psi$, there exist constants $\beta_{j}>0, j \in J_{2}$ such that

$$
\eta^{t}\left(\bar{x}, x^{0}\right) \mu_{j}^{0} \nabla g_{j}\left(x^{0}\right)+\beta_{j}\left\|\psi\left(\bar{x}, x^{0}\right)\right\|^{m} \leq 0, \quad j \in J_{2}
$$

As $\beta_{j}>0$,it follows that

$$
\eta^{t}\left(\bar{x}, x^{0}\right) \sum_{j \in J_{2}} \mu_{j}^{0} \nabla g_{j}\left(x^{0}\right) \leq 0
$$

Adding (5.3) and (5.4) and using $\lambda_{i}^{0} \geq 0, i=1,2, \ldots, p, \lambda^{0 t} \cdot e=1$, we obtain

$$
\eta^{t}\left(\bar{x}, x^{0}\right)\left[\sum_{i=1}^{p} \lambda_{i}^{0} \nabla f_{i}\left(x^{0}\right)+\sum_{j=1}^{q} \mu_{j}^{0} \nabla g_{j}\left(x^{0}\right)\right]<0
$$

which contradicts (3.2). Therefore,

$$
L\left(\bar{x}, \mu_{J_{1}}^{0}\right) \nless L\left(x^{0}, \mu_{J_{1}}^{0}\right)+c\left\|\psi\left(\bar{x}, x^{0}\right)\right\|^{m} .
$$

Again, since (3.3) holds and $x^{0} \in S$, we have

$$
f_{i}\left(x^{0}\right)+\sum_{j \in J_{1}} \mu_{j} g_{j}\left(x^{0}\right) \leq f_{i}\left(x^{0}\right)+\sum_{j \in J_{1}} \mu_{j}^{0} g_{j}\left(x^{0}\right) .
$$

This implies $L\left(x^{0}, \mu_{J_{1}}^{0}\right) \nless L\left(x^{0}, \mu_{J_{1}}\right)$.

Thus, $\left(x^{0}, \mu_{J_{1}}^{0}\right)$ is a mixed saddle point of order $m$ with respect to a nonlinear function $\psi$ for the partial vector Lagrangian.

Theorem 5.2 If $\left(x^{0}, \mu_{J_{1}}^{0}\right)$ is a mixed saddle point of order $m$ with respect to a nonlinear function $\psi$ for the partial vector Lagrangian, then $x^{0}$ is a strict minimizer of order $m$ with respect to the same $\psi$ for (MOP). 
Proof From the hypothesis $L\left(x^{0}, \mu_{J_{1}}^{0}\right) \nless L\left(x^{0}, \mu_{J_{1}}\right)$, we have

$$
\sum_{j \in J_{1}}\left(\mu_{j}^{0}-\mu_{j}\right) g_{j}\left(x^{0}\right) \nless 0 .
$$

Taking $\mu_{j}=0, j \in J_{1}$ in the above inequality, we obtain

$$
\sum_{j \in J_{1}} \mu_{j}^{0} g_{j}\left(x^{0}\right) \geq 0 .
$$

For $x^{0} \in S$ and the fact that $\mu_{J_{1}}^{0} \in R_{+}^{\left|J_{1}\right|}$, we have

$$
\sum_{j \in J_{1}} \mu_{j}^{0} g_{j}\left(x^{0}\right) \leq 0
$$

From above two inequalities, we have

$$
\sum_{j \in J_{1}} \mu_{j}^{0} g_{j}\left(x^{0}\right)=0 .
$$

Contrary to the result of the theorem, assume that $x^{0}$ is not a strict minimizer of order $m$ with respect to $\psi$ for (MOP). Then, for every $c \in \operatorname{int} R_{+}^{p}$, there exists an $\bar{x} \in S$ such that

$$
f(\bar{x})<f\left(x^{0}\right)+c\left\|\psi\left(\bar{x}, x^{0}\right)\right\|^{m}
$$

For any $\mu_{J_{1}}^{0} \in R_{+}^{\left|J_{1}\right|}$ and $\bar{x} \in S$, we have $\mu_{J_{1}}^{0} g_{J_{1}}(\bar{x}) \leq 0$.

Now,

$$
\begin{aligned}
L\left(\bar{x}, \mu_{J_{1}}^{0}\right)-c\left\|\psi\left(\bar{x}, x^{0}\right)\right\|^{m} & =f(\bar{x})+\mu_{J_{1}}^{0} g_{J_{1}}(\bar{x})-c\left\|\psi\left(\bar{x}, x^{0}\right)\right\|^{m} \\
& <f\left(x^{0}\right)+\sum_{j \in J_{1}} \mu_{j}^{0} g_{j}\left(x^{0}\right) \quad(\text { using (5.5) and (5.6)) } \\
& =L\left(x^{0}, \mu_{J_{1}}^{0}\right) .
\end{aligned}
$$

Therefore, $L\left(\bar{x}, \mu_{J_{1}}^{0}\right)-c\left\|\psi\left(\bar{x}, x^{0}\right)\right\|^{m}<L\left(x^{0}, \mu_{J_{1}}^{0}\right)$, which contradicts (5.2).

\section{An equivalent vector optimization problem}

In this section, we introduce an equivalent vector optimization problem (EVP) corresponding to (MOP) and prove that the problem of finding strict minimizers of order $m$ with respect to a nonlinear function $\psi: S \times S \rightarrow R^{n}$ for (MOP) reduces simply to the problem of finding strict minimizers for (EVP).

Let $x^{0}$ be any given feasible solution in (MOP). We consider the following equivalent vector optimization problem (EVP) given by

$$
\begin{aligned}
& \operatorname{minimize}\left(f_{1}\left(x^{0}\right)+\nabla f_{1}\left(x^{0}\right) \eta\left(x, x^{0}\right), \ldots, f_{p}\left(x^{0}\right)+\nabla f_{p}\left(x^{0}\right) \eta\left(x, x^{0}\right)\right) \\
& \text { subject to } g_{j}\left(x^{0}\right)+\nabla g_{j}\left(x^{0}\right) \eta\left(x, x^{0}\right) \leq 0, \quad j=1,2, \ldots, q,
\end{aligned}
$$


where $f_{i}, i=1,2, \ldots, p$ and $g_{j}, j=1,2, \ldots, q$ are defined as in (MOP). $\eta: S \times S \rightarrow R^{n}$ satisfies Assumption $C$ [12].

Let $D\left(x^{0}\right)=\left\{x \in X: g_{j}\left(x^{0}\right)+\nabla g_{j}\left(x^{0}\right) \eta\left(x, x^{0}\right) \leq 0, j=1,2, \ldots, q\right\}$ denote the set of all feasible solutions of (EVP).

Theorem 6.1 Let $x^{0}$ be a strict minimizer of order $m$ with respect to a nonlinear function $\psi: S \times S \rightarrow R^{n}$ for (MOP). Assume that Slater's constraint qualification (SCQ) holds at $x^{0}$, then $x^{0}$ is a strict minimizer in the equivalent vector optimization problem (EVP).

Proof Assume $x^{0}$ is a strict minimizer of order $m$ with respect to a nonlinear function $\psi: S \times S \rightarrow R^{n}$ for (MOP) and (SCQ) is satisfied at $x^{0}$; therefore, necessary optimality conditions (3.2)-(3.4) hold. Suppose $x^{0}$ is not a strict minimizer in (EVP). Then there exists $\bar{x}$ feasible for (EVP) such that for $i=1,2, \ldots, p$,

$$
f_{i}\left(x^{0}\right)+\nabla f_{i}\left(x^{0}\right) \eta\left(\bar{x}, x^{0}\right)<f_{i}\left(x^{0}\right)+\nabla f_{i}\left(x^{0}\right) \eta\left(x^{0}, x^{0}\right) .
$$

Since $\eta$ satisfies Assumption $C$, therefore $\eta\left(x^{0}, x^{0}\right)=0$ for $x^{0} \in S$, the above set of inequalities reduces to

$$
\nabla f_{i}\left(x^{0}\right) \eta\left(\bar{x}, x^{0}\right)<0, \quad i=1,2, \ldots, p
$$

Using $\lambda_{i}^{0} \geq 0, i=1,2, \ldots, p$, we have

$$
\sum_{i=1}^{p} \lambda_{i}^{0} \nabla f_{i}\left(x^{0}\right) \eta\left(\bar{x}, x^{0}\right)<0
$$

Since $\mu_{j} \geq 0, j=1,2, \ldots, q$, therefore for $\bar{x} \in D\left(x^{0}\right)$, it follows that

$$
\mu_{j}^{0} g_{j}\left(x^{0}\right)+\mu_{j}^{0} \nabla g_{j}\left(x^{0}\right) \eta\left(\bar{x}, x^{0}\right) \leq 0, \quad j=1,2, \ldots, q .
$$

Using (3.3), we have

$$
\sum_{j=1}^{q} \mu_{j}^{0} \nabla g_{j}\left(x^{0}\right) \eta\left(\bar{x}, x^{0}\right) \leq 0 .
$$

Adding (6.2) and (6.3), we get a contradiction to (3.2).

Theorem 6.2 Let $x^{0}$ be a strict minimizer in the equivalent vector optimization problem (EVP). Further assume that $f_{i}, i=1,2, \ldots, p$ are strongly pseudoinvex type I of order $m$ and $g_{j}, j=1,2, \ldots, q$ are strongly invex of order $m$ with respect to the same $\eta$ and $\psi$, then $x^{0}$ is a strict minimizer of order $m$ in the original vector optimization problem (MOP).

Proof Clearly, $x^{0}$ is feasible for (MOP). First, we will show that any feasible point in (MOP) is also a feasible point in (EVP), that is, we will show that $S \subseteq D\left(x^{0}\right)$. Let $x \in S$ and $g_{\text {, }}$, $j=1,2, \ldots, q$ be strongly invex of order $m$ with respect to $\eta$ and $\psi$ on $X$. Therefore, for some $k_{j}>0, j=1,2, \ldots, q$, we have

$$
g_{j}(x)-g_{j}\left(x^{0}\right) \geq \nabla g_{j}\left(x^{0}\right) \eta\left(x, x^{0}\right)+k_{j}\left\|\psi\left(x, x^{0}\right)\right\|^{m}, \quad j=1,2, \ldots, q .
$$


As $x \in S$ and $k_{j}>0, j=1,2, \ldots, q$, the above inequalities lead to

$$
0 \geq g_{j}\left(x^{0}\right)+\nabla g_{j}\left(x^{0}\right) \eta\left(x, x^{0}\right), \quad j=1,2, \ldots, q,
$$

that is, $x \in D\left(x^{0}\right)$. Hence, $S \subseteq D\left(x^{0}\right)$.

Now, suppose that $x^{0}$ is not a strict minimizer of order $m$ in (MOP). Then, for $\beta_{i}>0$, $i=1,2, \ldots, p$, there exists some $\bar{x} \in S$ such that

$$
f_{i}(\bar{x})<f_{i}\left(x^{0}\right)+\beta_{i}\left\|\psi\left(\bar{x}, x^{0}\right)\right\|^{m}, \quad i=1,2, \ldots, p
$$

Since $f_{i}, i=1,2, \ldots, p$ are strongly pseudoinvex type I of order $m$ with respect to $\eta$ and $\psi$ on $S$, we have

$$
\nabla f_{i}\left(x^{0}\right)^{t} \eta\left(\bar{x}, x^{0}\right)<0, \quad i=1,2, \ldots, p .
$$

Since $\eta\left(x^{0}, x^{0}\right)=0$, we can rewrite the above set of inequalities as

$$
f_{i}\left(x^{0}\right)+\nabla f_{i}\left(x^{0}\right) \eta\left(\bar{x}, x^{0}\right)<f_{i}\left(x^{0}\right)+\nabla f_{i}\left(x^{0}\right) \eta\left(x^{0}, x^{0}\right), \quad i=1,2, \ldots, p,
$$

which contradicts that $x^{0}$ is a strict minimizer for (EVP).

\section{Competing interests}

The authors declare that they have no competing interests.

\section{Authors' contributions}

All authors contributed equally and significantly in writing this article. All authors read and approved the final manuscript.

\section{Author details}

${ }^{1}$ Department of Mathematics, University of Delhi, Delhi, 110007, India. ${ }^{2}$ Department of Operational Research, University of Delhi, Delhi, 110007, India.

\section{Acknowledgements}

The authors would like to thank Prof. Davinder Bhatia (Retd.) and Dr. Pankaj Gupta, Department of Operational Research, University of Delhi, for their keen interest and continuous help throughout the preparation of this article.

\section{Received: 19 April 2012 Accepted: 6 January 2013 Published: 24 January 2013}

\section{References}

1. Weir, T, Mond, B: Pre-invex functions in multiple objective optimization. J. Math. Anal. Appl. 136, $29-38$ (1988)

2. Sawaragi, Y, Nakayama, H, Tanino, T: Theory of Multiobjective Optimization. Academic Press, Orlando (1985)

3. Cromme, L: Strong uniqueness: a far reaching criterion for the convergence of iterative procedures. Numer. Math. 29, 179-193 (1978)

4. Studniarski, M: Sufficient conditions for the stability of local minimum points in nonsmooth optimization. Optimization 20, 27-35 (1989)

5. Auslender, A: Stability in mathematical programming with non-differentiable data. SIAM J. Control Optim. 22, 239-254 (1984)

6. Ward, DE: Characterization of strict local minima and necessary conditions for weak sharp minima. J. Optim. Theory Appl. 80, 551-571 (1994)

7. Jimenez, B: Strict efficiency in vector optimization. J. Math. Anal. Appl. 265, 264-284 (2002)

8. Bhatia, G: Optimality and mixed saddle point criteria in multiobjective optimization. J. Math. Anal. Appl. 342(1), 135-145 (2008)

9. Sahay, RR, Bhatia, G: Characterizations for the set of higher order global strict minimizers. J. Nonlinear Convex Anal. (to appear)

10. Lin, GH, Fukushima, M: Some exact penalty results for non-linear programs and mathematical programs with equilibrium constraints. J. Optim. Theory Appl. 118,67-80 (2003)

11. Mangasarian, OL: Nonlinear Programming. Classical Appl. Math., vol. 10. SIAM, Philadelphia (1994) (corrected reprint of the 1969 original)

12. Mohan, SR, Neogy, SK: On invex sets and preinvex functions. J. Math. Anal. Appl. 189, 901-908 (1995) 
doi:10.1186/1029-242X-2013-31

Cite this article as: Bhatia and Sahay: Strict global minimizers and higher-order generalized strong invexity in multiobjective optimization. Journal of Inequalities and Applications 2013 2013:31.

Submit your manuscript to a SpringerOpen ${ }^{\circ}$ journal and benefit from:

- Convenient online submission

- Rigorous peer review

- Immediate publication on acceptance

Open access: articles freely available online

- High visibility within the field

- Retaining the copyright to your article

Submit your next manuscript at $\gg$ springeropen.com 